\title{
Linx
}

LinX Revue des linguistes de l'université Paris X Nanterre

$54 \mid 2006$

La cause : approche pluridisciplinaire

\section{Aperçu de l'évolution des conceptions de la cause dans les sciences et particulièrement dans les sciences économiques}

Bruno Lefebvre

\section{OpenEdition}

Journals

Édition électronique

URL : http://journals.openedition.org/linx/496

DOI : $10.4000 / \operatorname{linx} .496$

ISSN : 2118-9692

Éditeur

Presses universitaires de Paris Nanterre

\section{Édition imprimée}

Date de publication : 1 juin 2006

Pagination : 19-25

ISSN : 0246-8743

\section{Référence électronique}

Bruno Lefebvre, «Aperçu de l'évolution des conceptions de la cause dans les sciences et particulièrement dans les sciences économiques », Linx [En ligne], 54 | 2006, mis en ligne le 01 août 2007, consulté le 04 mai 2019. URL : http://journals.openedition.org/linx/496 ; DOI : 10.4000/linx.496 


\title{
Aperçu de l'évolution des conceptions de la cause dans les sciences et particulièrement dans les sciences économiques
}

\author{
Bruno Lefebvre, Université Paris $X$ - Nanterre \\ Sciences économiques \\ Vice-président chargé des études et de la vie universitaire
}

Par ses sens et par son intelligence, l'Homme ne peut appréhender qu'une partie du monde réel. Il lui faut donc construire une stratégie qui lui permette de rendre intelligibles, l'origine, la constitution et le devenir de ce qui est. Selon les philosophes grecs classiques, cela doit être l'ambition majeure de la pensée humaine. Partant de l'affirmation de Platon dans le Timée selon laquelle «tout ce qui naît naît nécessairement d'une cause », cette stratégie passe nécessairement par la recherche des causes.

$\mathrm{Au}$ cours du temps, cette dernière a fait l'objet de nombreuses critiques, certaines pouvant laisser croire qu'il convenait de l'abandonner. Mais il convient d'en distinguer différents types et si l'on en exclut certains, la recherche de la causalité demeure essentielle à la démarche scientifique. Le sujet de la «cause » doit donc nous interpeller, en tant que chercheurs, chacun dans son champ disciplinaire, sur ses pratiques, ses méthodes... Dans un premier temps, je ferai quelques rappels généraux au travers d'une brève histoire de la notion de "causalité » pour, dans un deuxième temps, recentrer mon propos sur les sciences sociales avant de me focaliser, pour terminer, sur la science économique, ma propre discipline. 


\section{Une brève histoire de la notion de « causalité "}

La première conception de la causalité, celle que l'on doit à Platon et à ses successeurs, relève de la métaphysique. En effet, les philosophes grecs classiques lient l'idée de «cause» à l'origine des choses. La «cause» est le principe d'explication de la création. Il s'agit donc d'une cause première, celle-ci pouvant être, selon les auteurs, de nature divine.

Bien entendu, la démarche scientifique exclut ce type de causalité. Les sciences partent de l'hypothèse selon laquelle les constituants du monde matériel existent, et qu'elles ont à en étudier les propriétés et les transformations. Et ces transformations ne peuvent être dues à une intervention divine, mais à des causes nécessaires, mécaniques, «errantes». Enfin si les sciences partent du principe que «tout ce qui naît, nait nécessairement d'une cause », elles en tirent le corollaire selon lequel le monde est gouverné par des lois telles que «lorsque les mêmes conditions sont réalisées à deux instants différents, en deux lieux différents de l'espace, les même phénomènes se reproduisent transposés seulement dans le temps et dans l'espace »1.

C'est dans la mécanique newtonienne puis, plus généralement, dans la physique classique que l'explication causale scientifique s'impose au XVII siècle. Mais simultanément apparaissent les premières critiques. Elles viennent de Malebranche (1638-1715) : «Si Dieu est liberté et que ses volontés soient inscrutables par la raison humaine, nos causes ne sont que des fictions forgées par notre esprit... Certes, nous observons un parallélisme entre les lois mathématiques et des vérités d'expérience... Cette cohérence entre l'ordre des déductions mathématiques et celui des observations empiriques peut être voulue et instituée par Dieu sans exprimer pour autant une affinité ou une identité entre l'ordre de nos idées et celui des choses $»^{2}$. Cette objection sera reprise par David Hume en 1748 dans L'enquête sur l'entendement bumain. Pour lui, les relations causales existent, mais elles relèvent d'une observation ou d'une expérience récurrente et non d'une relation rationnelle et nécessaire entre cause et effet telle que définie précédemment. C'est Emmanuel Kant, dans la Critique de la raison pure parue en 1781 qui réhabilite l'idée de «cause » en expliquant que si la connaissance commence avec l'expérience elle ne dérive pas nécessairement de celle-ci.

De nouvelles critiques apparaissent à partir du XIX ${ }^{e}$ siècle. Du côté des sciences sociales, Auguste Comte, puis ceux qui se réclament de sa pensée reprennent et développent les critiques énoncées par David Hume. Du côté des sciences physiques, elles sont liées au développement des probabilités, mais elles ne portent pas tant sur le principe de causalité que sur l'usage qui en est fait en vue de réaliser des prédictions. Cet usage ne serait possible que si deux conditions sont réunies. La première est l'existence d'un outil mathématique et de calcul apte à réaliser ces prédictions. La seconde est la connaissance entière des conditions initiales du phénomène décrit par la relation causale.

\footnotetext{
1 Paul Painlevé, 1922, Les axiomes de la mécanique, Examen critique; Gauthier-Villars cité par Bertrand Saint-Sernin dans l'article " Causalité », Encyclopaedia Universalis.

${ }^{2}$ Cité par Bertrand Saint-Sernin dans l'article « Causalité », Encyclopaedia Universalis.
} 
Aujourd'hui, si l'on suit Karl Popper (1902 -1994), «nous ne devons jamais renoncer à nos essais en vue d'expliquer par un lien causal toute espèce d'évènement que nous pouvons décrire $»^{3}$. Toutefois il convient de préciser la notion de «causalité scientifique ». Comme nous l'avons vu, nous devons exclure la cause première, mais aussi la causalité substantielle, toutes les deux étant en opposition avec le principe de réfutabilité au sens de Popper. Mais il convient également d'exclure d'autres formes de causalité comme la causalité circulaire qui explique un phénomène par un autre et ce dernier par le premier.

Refuser les causalités circulaires signifie que l'on accepte que l'explication d'un phénomène initial ne soit pas donnée par la science. Ce phénomène initial devient un présupposé. Partant de celui-ci, nous avons une chaine de causalités : A est la cause de $\mathrm{B}$ qui est lui-même la cause de $\mathrm{C}$ et ainsi de suite. Dans une telle chaîne, pour admettre scientifiquement que $\mathrm{A}$ est la cause de $\mathrm{B}$, il est d'abord nécessaire de rendre intelligible le cheminement qui conduit du premier au second puis de pouvoir le reproduire. Cette nécessité de le reproduire, au moins par l'esprit, suppose la cohérence du raisonnement qui conduit du premier au second. Cette cohérence peut être assurée par le recours aux mathématiques, cohérentes en elles-mêmes et de ce fait supérieures dans cette mission à un discours littéraire. Toutefois, le fait de ne pas pouvoir recourir à un discours formel n'empêche pas la recherche de relations causales. C'est le cas dans de nombreux domaines des sciences sociales.

\section{La causalité dans les sciences sociales}

Le rapport qu'entretiennent les sciences sociales avec la notion de «cause » est ambigu. D'une part, celles-ci refusent la notion de «cause» s'inscrivant dans la continuité des critiques posées par David Hume et reprises par Auguste Comte. Mais d'autre part, le développement des recherches empiriques s'est opéré par le recours à un langage causal et par le développement d'instruments d'analyse causale rigoureux.

Le refus de la notion de «cause » telle qu'elle est utilisée dans les sciences de la nature et en particulier dans les sciences physiques a deux origines. La première tient au caractère spécifique de l'objet étudié par les sciences sociales, qui exclut le déterminisme. En effet, les évènements qui surviennent dans l'espace social sont au moins partiellement le fait d'acteurs capables de décider, d'anticiper, de réagir, etc. acteurs ayant donc un libre arbitre. Or il ne peut y avoir libre arbitre et déterminisme strict. Cet argument est d'ailleurs défendu par Emmanuel Kant dans la Critique de la raison pratique. La seconde tient au fait que ce sont des relations statistiques qui sont mises en évidence ou observées. Ces relations peuvent-elles être pour autant des relations de cause à effet ? La réponse a priori est non puisque d'une relation statistique on peut aussi bien déduire que A est la cause de B ou que B est la cause de A.

Si l'idée de «cause» ne peut être appliquée dans les sciences sociales, il faut donc recourir à d'autres modes d'explication. Le premier possible est le fonctionnalisme. Il part de l'hypothèse que la société est une totalité et que ses composantes ne peuvent être étudiées que dans leurs rapports à cette totalité, c'est-à-dire par leur fonction. Le

\footnotetext{
${ }^{3}$ Karl Popper, 1934, La Logique de la découverte scientifique, Paris, Payot 1984, p. 54.
} 
second est la compréhension, puisque les phénomènes sociaux contrairement aux phénomènes naturels sont immédiatement signifiants.

C’est Émile Durkheim (1858-1917) qui dans Les Règles de la méthode sociologique ouvrage publié en 1895, pose les principes de l'utilisation des relations causales en sciences sociales. Tout d'abord, les faits sociaux doivent être considérés par ceux qui les étudient comme des objets dont ils sont extérieurs. Ensuite, compte tenu du fait que l'action d'un phénomène social $\mathrm{A}$ sur un autre phénomène social $\mathrm{B}$ se confond avec les actions de nombreux autres phénomènes sociaux sur ce même phénomène social $\mathrm{B}$, il est difficile d'obtenir directement une explication du phénomène social $\mathrm{B}$. Il faut donc adopter une démarche déductive comme dans les sciences de la nature. Cela implique de construire des relations causales dans lesquelles la cause d'un fait social est toujours un autre fait social. Ces relations causales sont des hypothèses qui doivent être ensuite vérifiées. Cela peut passer par le recours à la statistique et aux méthodes mathématiques d'analyse causale.

Dans les sciences sociales, les sciences économiques occupent une place particulière. D'une part, elles se sont développées de façon autonome, avant les autres sciences sociales, demeurées étroitement liées à la philosophie. D'autre part, elles ont revendiqué très tôt leur développement sur le modèle des sciences physiques.

\section{La causalité dans les sciences économiques}

C'est à partir du XVII" siècle que la pensée économique connait un développement important, stimulée par les nouveaux courants de la pensée philosophique.

Francis Bacon (1561-1626) est le premier auteur qui sépare clairement les sciences de la religion et qui contribue à leur donner un espace avec des règles qui leur soient propres. Selon lui, la nature est régie par des lois et l'objectif ultime de la recherche scientifique est la découverte de ces lois, qui doivent être exprimées sous la forme de relations causales. De plus, cette recherche passe par l'induction et l'expérimentation. Mais les principes dégagés pour l'étude de la nature peuvent également s'appliquer à l'analyse des problèmes éthiques, psychologiques et sociaux.

Le premier philosophe à essayer d'appliquer la méthode prônée par Francis Bacon à l'analyse des faits sociaux est Thomas Hobbes (1623-1687) dans Le Léviathan publié en 1651. Selon lui, les besoins et la peur sont les causes premières du comportement humain. Le désir d'être en sécurité amène les hommes à accepter un contrat social. Ainsi, selon lui, c'est un objectif utilitaire qui fonde la vie en communauté. Il définit alors la raison comme l'instrument qui permet de créer les moyens propres à atteindre les objectifs utilitaires et donne ainsi une première forme au comportement rationnel. Enfin, il redéfinit les «lois de la nature» comme les lois qui régissent les actions d'un être idéalement raisonnable. C'est le début de la philosophie utilitariste dont se réclamera Adam Smith (1723-1790), philosophe anglais présenté très souvent comme le " père » de la science économique.

Cependant, le premier économiste qui cherche à appliquer les principes de Francis Bacon est William Petty (1623-1687). Cela le conduit à une formulation des problèmes économiques en termes de relations entre des grandeurs mesurables. Il est à ce titre l'un des précurseurs de l'économie quantitative. A partir de ce moment, 
l'établissement de relations mathématiques entre des grandeurs économiques mesurables semble permettre de découvrir des propositions fiables basées sur l'expérience et l'observation.

Une nouvelle étape est franchie en particulier avec Dudley North (1641-1691) qui centre l'étude des phénomènes économiques sur ceux qui peuvent être interprétés en termes de mécanismes d'autorégulation. La pensée économique anglaise se développe alors selon une conception libérale, l'État n'ayant pas à intervenir dans l'économie puisque celle-ci peut s'autoréguler. En revanche, sur le continent, la pensée mercantiliste continue de prédominer. Il faut attendre les physiocrates français au XVIII siècle pour découvrir une première version du libéralisme.

Celui-ci triomphe avec la diffusion de l'économie utilitariste à la fin du XVIII et tout au long du XIXe siècle. La méthodologie utilitariste telle qu'elle est employée par les sciences économiques est élaborée par Jeremy Bentham (1748-1832), philosophe anglais contemporain d'Adam Smith. Suivant Thomas Hobbes, il précise que pour apprécier la valeur d'une action il faut en étudier les conséquences en termes de plaisir et de douleur. Il va plus loin en mesurant l'utilité d'une action par le supplément de bonheur ou la réduction de douleur qu'elle apporte dans le système d'échanges des plaisirs et des douleurs tel qu'il existait avant cette action. Toutefois, peut-on effectivement mesurer objectivement ces quantités qui relèvent du subjectif ? Selon lui cette question est similaire à celle qui se pose à la physique qui doit mesurer objectivement des grandeurs qui sont d'abord perçues sous une forme qualitative comme la chaleur ou la vitesse. La même réponse peut lui être apportée. Cela a deux conséquences. Premièrement, il n'y a pas lieu de distinguer les méthodes employées par les sciences sociales de celles auxquelles recourent les sciences de la nature. Deuxièmement, il est possible d'introduire en économie les méthodes évoluées du raisonnement hypothétique.

Le premier auteur à avoir appliqué à l'économie les méthodes de l'utilitarisme énoncées par Jeremy Bentham est David Ricardo (1772-1823), même s'il n'a jamais explicité ses méthodes d'analyse. D'abord, il suppose que les relations sociales montrent une régularité comparable aux lois de causalité découvertes par les sciences de la nature. Ensuite, il considère que l'on ne peut compter sur la raison pour établir et perpétuer un ordre satisfaisant dans les relations économiques. On ne peut pas davantage compter sur des forces métaphysiques comme la main invisible d'Adam Smith. Cet ordre ne peut venir que du jeu de certaines forces qui aboutissent à coordonner le nombre infini des décisions humaines. Ces forces sont celles de la concurrence qui jouent dans l'économie un rôle similaire à celui des forces mécaniques dans le monde cosmique. L'étude du fonctionnement de l'économie peut donc être séparée de toute considération politique, morale ou sociologique. Comme dans le modèle newtonien, il est fait abstraction du temps. Comme dans le modèle newtonien, le système englobe des corps qui sont des agrégats d'unités élémentaires invisibles, l'unité de valeur d'échange. Les corps peuvent alors être décrits en un dénominateur commun permettant l'emploi des mathématiques pour l'analyse du comportement du système. Cette approche de l'économie va dominer en Angleterre pendant tout le $\mathrm{XIX}^{\mathrm{e}}$ siècle.

Sur le continent, et en particulier en France, les économistes refusent la démarche ricardienne et préfèrent s'inscrire dans une démarche plus «baconienne » 
fondée sur l'observation et l'expérience. De même refusent-ils d'appliquer les méthodes mathématiques. Cependant, dans le même temps, des mathématiciens s'intéressent aux questions économiques comme Antoine Augustin Cournot (18011877). Il sera l'un des précurseurs de l'économie mathématique.

Les méthodes hypothétiques sont également rejetées par les auteurs allemands encore sous l'influence de la philosophie d'Emmanuel Kant. Ceux-ci utilisent des procédés plus intuitifs pour définir leurs thèses et leurs concepts. A partir du début du XIX ${ }^{e}$ siècle se développe l'école historique allemande. Influencée par la philosophie de Hegel, elle critique à la suite de Bruno Hildebrand (1812-1878) l'immuabilité des lois naturelles et préconise leur remplacement par des lois de développement social, lois mises en évidence selon une démarche inductive. Certains de ses membres iront même plus loin en limitant le rôle de l'économiste à la simple description de cadres historiques. Cette opposition de l'école historique allemande aux méthodes hypothétiques aboutit en 1883 «au conflit des méthodes» avec la publication de l'ouvrage de Karl Menger (1840-1921) Recherche sur la méthode des sciences sociales et de l'économie politique en particulier.

Selon ce dernier, l'économie conçue comme une science exacte a pour mission de mettre en évidence certaines régularités de séquences et cela ne peut se faire qu'en réduisant les phénomènes sociaux à quelques éléments quantitatifs simples mesurés selon des normes appropriées. Il faut donc avoir une image générale et abstraite des relations causales entre quelques grandeurs économiques. L'économie peut même employer des concepts très abstraits n'ayant pas nécessairement de contrepartie dans la réalité, mais qui peuvent être associés les uns aux autres par une logique appropriée.

A la fin du $\mathrm{XIX}^{\mathrm{e}}$ siècle et au début du $\mathrm{XX}^{\mathrm{e}}$ siècle deux conceptions vont à nouveau s'affronter. D'un côté, on retrouve les tenants d'un raisonnement hypothétique évolué, certains avec des hypothèses très abstraites dans la lignée des économistes mathématiciens de l'école de Lausanne ou des marginalistes américains comme John Bates Clark (1847-1938), d'autres les refusant comme Alfred Marshall (1842-1924) en Angleterre. De l'autre côté, des économistes, influencés par Durkheim, essentiellement français comme François Simiand (1873-1935), insistent sur la nécessité d'introduire des éléments de sociologie dans l'analyse économique. Cette dernière tendance débouche sur la sociologie économique qui définit des relations causales entre des ensembles bien circonscrits de phénomènes économiques et les évènements sociaux concomitants. Cette opposition existe toujours, elle s'est même renforcée. Les tenants de la première approche constituent ce que l'on peut appeler «l'école orthodoxe» et ceux de la seconde ce que l'on peut appeler "l'école hétérodoxe ».

\section{Conclusion}

Si l'on excepte l'école historique allemande et la sociologie économique, les sciences économiques se sont développées selon le modèle des sciences physiques. On peut alors se demander si les relations causales en économie sont équivalentes aux relations causales en physique. La réponse est plutôt négative pour cinq raisons : 
Aperçu de l'évolution des conceptions de la cause dans les sciences...

- les concepts ne sont pas toujours parfaitement définis, si bien que la cause et l'effet d'une relation ne le sont pas non plus ;

- si le pouvoir prédictif d'une relation causale n'est pas réfuté, il n'en demeure pas moins qu'en raison de l'irréalisme des hypothèses sur laquelle elle repose, elle ne permet pas d'expliquer le phénomène étudié ;

- les lois que traduisent les relations causales dans les faits sont datées ;

- certaines des causalités sont circulaires ;

- enfin, les représentations de l'économie qu'ont les agents déterminent leurs actions et donc modifient la réalité économique. 
